\title{
Reading errors made by children with low vision
}

* Graeme Douglas ${ }^{1}$, Mick Grimley ${ }^{2}$, Mike McLinden ${ }^{1}$ and Linda Watson ${ }^{1}$

${ }^{1}$ Visual Impairment Centre for Teaching and Research (VICTAR), School of Education, University of Birmingham, Birmingham B15 2TT, UK, and 'Education Department, University of Canterbury, Private Bag 4800, Christchurch, New Zealand.

\begin{abstract}
Previous research has shown that, on average, children with low vision lag their sighted peers in general reading development (in terms of speed, accuracy and comprehension). This study sought to examine this apparent lag by comparing the reading profiles of 25 normally sighted readers (mean age 8 years 8 months) with 25 low vision readers. The children were tested using a reading test (the Neale Analysis of Reading Ability, NARA) and were matched on the reading accuracy score produced by the test. Therefore in terms of the reading accuracy scores (and reading ages) derived from the NARA both groups were the same. The low vision readers were on average older than the normally sighted children (mean $=10$ years, 5 months). When the reading profile (i.e. accuracy, comprehension and speed) was examined in the same analysis no significant effect was revealed [d.f. $=1,48 ; F=0.05 ; p>0.1$ ], but a general lag for these children is suggested (in keeping with previous research). However, a closer analysis of the reading error profile revealed the most common reading errors made by all readers in the analysis were either mispronunciations or substitutions. The low vision readers were more prone to making substitution errors than mispronunciations and the reverse was true for normally sighted readers [d.f. $=1,48 ; F=7.1 ; p<0.05$ ]. This indicates that the reading strategies adopted by low vision readers may differ from those of normally sighted readers of the same apparent reading ability.
\end{abstract}

Keywords: reading, low vision, children

\section{Introduction}

A large amount of useful research has been carried out into the reading errors of normally sighted children. For example, Goodman (1967) carried out much of the initial work into reading errors, or 'miscues', and Leu (1982) provides a review. In addition, many published assessments evaluate reading errors, e.g. the Neale Analysis of Reading Ability (NARA) (e.g. Neale, 1997). NARA is a standardised assessment of oral reading for approximate age range 6-13 years. It provides three scores of reading performance - accuracy, comprehension and speed. While it does not provide standard scores for the types of errors made, it does provide diagnostic tests which categorise errors into mispronunciations, substitutions, refusals, additions, omissions and reversals.

Relatively little work has been carried out into the reading errors of children with low vision. However, Douglas et al. (2002) tested the reading of 476 children with low vision using an unmodified print version of the NARA. The data showed that the average reading ages for accuracy, comprehension and speed for the sample are generally below their chronological age when the comparison is made with their fully sighted peers. This finding was in keeping with general observations of teachers and existing literature (e.g. Tobin, 1993 in terms of speed; Gompel et al., 2002 in terms of 'decoding'). It appears that up to the age of approximately 7 years, the reading performance of children with low vision is in line with sighted peers but then begins to lag, and the size of this lag increases with age.

In the area of the psychophysics of reading, some research has been carried out investigating the eye movements of low vision readers (adults) by Legge et al. (1996) [e.g. they showed that the visual span was 
smaller and saccades were therefore shorter (in addition to fixations being longer) for low vision readers than normally sighted readers]. One of the few studies to investigate reading errors directly was carried out by Corley and Pring (1993). They examined the reading errors of nine partially sighted children (aged 5:8-8:7 years) in detail (repeated testing over a 7-month period). They found the errors made resembled those of fully sighted children of a younger age, but otherwise there were no categorical differences. However, with such a small sample of children it is possible that any such differences would be lost.

Similar studies to the one presented here have been carried out with deaf children. Conrad (1979) carried out a large-scale study into the reading attainment of deaf pupils which demonstrated a considerable lag in comparison with hearing pupils. Other researchers have found similar results and the concept of a 'reading plateau' for deaf pupils has been widely accepted (see Quigley and Paul, 1990 for a summary).

Following on from this work, Webster et al. (1981) analysed deaf children's patterns of errors during standard reading activities. Using a miscue analysis (Goodman, 1969), they uncovered several typical patterns in the deaf children's responses. A further study of deaf children's errors (Wood et al., 1981) used the Southgate (1962) test, which requires the pupils to insert a missing word. They tested 60 deaf children and 60 hearing children and compared their results. The average age of the deaf children was 11 years and the average age of the hearing children was 9 years. The average number of errors made by the deaf children was $47 \%$, and the average for the hearing children was $18 \%$. Analysis of the deaf children's errors showed a remarkable degree of consistency between them, but their choices were different from those made by the hearing children.

The conclusion drawn from these studies was that deaf and hearing children were using different strategies in approaching these reading tests. The tests were therefore sampling different behaviours in the two groups. The assumption that a deaf pupil aged 16 who achieves a reading age of 9 years, can be compared with a hearing pupil aged 9 years has been challenged on the basis that the scores may be achieved in a different way and that the older pupil will be more sophisticated emotionally and conceptually (Webster, 1986). This research served to highlight both the fact that deaf pupils' achievements in reading lagged behind those of hearing pupils and also the positive fact that an analysis of reading errors revealed creative strategies that the deaf pupils were bringing to the reading process.

\section{Aims}

The research sought to analyse whether low vision children adopted different reading strategies to normally sighted children who were matched in terms of reading age.

\section{Method}

\section{Participants}

Fully sighted participants were recruited from two classes in an urban primary school (year 3 and 5). Twentyfive children (aged 7:8-10:6 years; 11 females, 14 males) were tested using form 1 of the NARA. These children were matched with 25 children with low vision (see below) who had also been tested using the NARA. The children with low vision (aged 6:5-14:3 years; 11 females, 14 males) were selected from data collected for another project ( $n=231$, see Douglas et al., 2002). The children had a range of visual impairments, although all those in this study carried out the reading test without using a low vision aid (LVA). The children were educated in different mainstream schools in England.

\section{Matching participants}

Children were matched in terms of their reading accuracy scores as measured by the NARA (which generates a score ranging between 0 and 100 - higher scores indicate higher reading accuracy). A more detailed description of the NARA is presented in Douglas et al. (2002). The scores ranged from 34 to 83, and children were matched in 24 of 25 cases (in the case of one pair the difference was by a single mark, which was the 
closest match in the available data). Therefore in terms of the reading accuracy scores (and reading ages) derived from the NARA the two groups were the same. As far as the data would allow, children were also matched in terms of school placement (in 21 cases the visually impaired students attended mainstream schools, and in the remaining four cases they were also supported by a unit in a mainstream school), and gender (in eight cases the gender did not match). Within these criteria, if more than one visually impaired student matched, a random choice was made.

\section{Analysis}

Analyses were carried out which compared the reading characteristics of the two groups - normally sighted and low vision. This took three stages

A comparison of the ages of the two groups (ANOVA);

A comparison of the reading speed and comprehension of the two groups (ANOVA);

A comparison of the reading errors made by the two groups (ANOVA).

\section{Results and discussion}

\section{Comparison of the ages of the two groups}

The low vision readers were on average older than the normally sighted children (mean = 10 years, 5 months cf. mean $=8$ years, 8 months). This proved high statistical significance (d.f. $=1, F=10.9, p<0.005$ ). In line with the discussion presented in the introduction, this is in keeping with the specific findings of Douglas et al. (2002), and more general literature (e.g. Tobin, 1993; Gompel et al., 2002).

\section{Comparison of reading speed and comprehension of the two groups}

A one-way analysis of variance of group (low vision and normally sighted) with repeated measures of reading comprehension and reading speed was performed on the data. No significant effects were found for a main effect of visual impairment (d.f. $=1, F=0.005, p=0.945$ ) or an interaction of visual impairment and type of reading measure (d.f. $=1,48, F=0.004, p=0.95$ ). This suggests that the low vision children's reading lags in terms of accuracy, comprehension and speed in equal amounts. Therefore, in terms of this analysis, the two groups cannot be distinguished, i.e. there is a general lag.

\section{Comparison of reading errors made by the two groups}

The previous analysis implies that children with low vision simply read in the same way as younger normally sighted children, i.e. the general lag position. As described above, the NARA categorises reading errors into one of six categories. However, the majority of reading errors fall into the category of substitution and mispronunciation (74\% of errors in the case of normally sighted readers; $82 \%$ of errors in the case of low vision readers). For this reason it was decided to carry out an analysis which focused upon these errors only. A one way analysis of variance of group (low vision and normally sighted) with repeated measures of reading error subscores (substitutions and mispronunciations) was performed on the data. A significant interaction of reading error and type of reader was found ( $d . f .=1,48 ; F=7.1 ; p<0.05$ ). The low vision readers were more prone to making substitution errors than mispronunciations (mean of 8.8 and 5.2 errors per reader respectively) and the reverse was true for normally sighted readers (mean of 6.1 and 6.8 errors per reader respectively) - see Figure 1 . However, neither direct comparison is significant on its own (i.e. mispronunciation for low vision vs normally sighted; substitution for low vision vs normally sighted). 


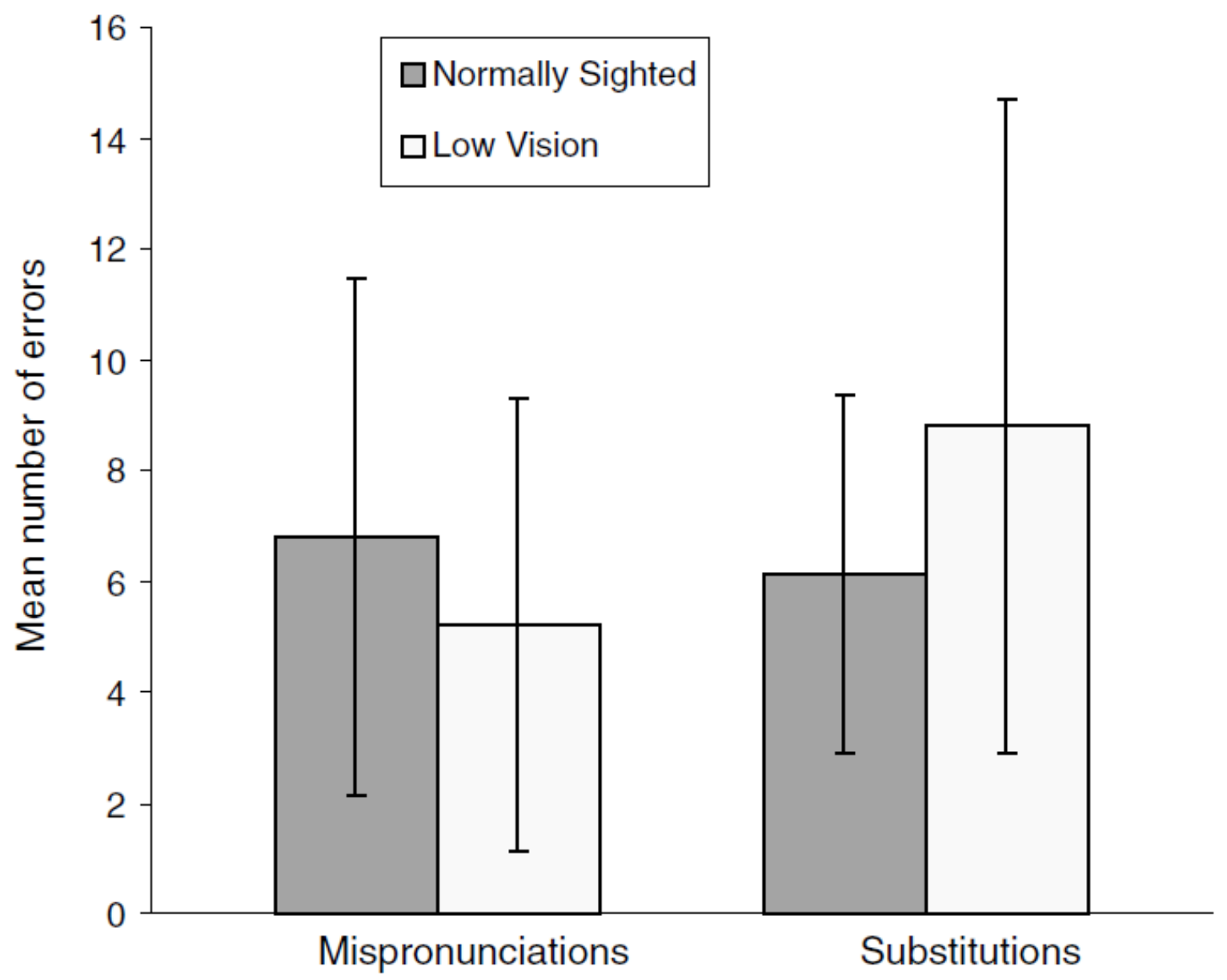

Figure 1. The reading error profile of readers $(n=50)$ illustrates that those with low vision tend to make more substitution errors than mispronunciation errors when compared with a matched sample of normally sighted readers.

\section{Additional description of reading errors}

A closer look at the individual participant data suggests that the effect amongst the low vision readers was to make more substitution errors than mispronunciation errors. Of the 25 low vision readers, all but three showed more substitution than mispronunciation errors. In the case of the normally sighted readers no pattern was observed (12 participants with more mispronunciations, 13 with more substitutions).

\section{Discussion and conclusions}

The findings indicate that low vision and normally sighted readers matched on reading comprehension, speed and accuracy appear to show subtle differences in their reading processes. Low vision readers were shown to be more prone to making substitution errors. The pattern of errors displayed by low vision readers suggests that they may be relying on different strategies to decode the text. There may be two explanations for this, which may well coexist. First, it might be expected that a student who is on average 2 years older and finding it difficult to see the print may be more prone to 'guessing' a semantically appropriate word. This could be broadly called the 'semantic top down' explanation. Secondly, low vision readers may mistakenly identify words that look similar to one another in terms of their orthographic pattern. This could be broadly called an 'orthographic top down' explanation. Future research might carry out a closer analysis of the errors themselves to determine if either (or both) of these explanations has any validity. Future research might also look more carefully at the natures and severities of the visual impairments. For example, it might be 
anticipated that children with a particular eye condition such as nystagmus might be prone to particular errors. Another line of future research might explore whether older readers generally (normally sighted and low vision) are more prone to 'guessing' a semantically appropriate word in circumstances where the text is difficult to access.

Reading research into normally sighted readers suggest that readers who rely on context when decoding text are poorer readers than those readers who rely on phonological cues or orthographic patterns to decode text (Nicholson et al., 1988; Laing, 2002), and as text becomes more complex, phonological and orthographic strategies prove far more effective (Laing, 2002). It is possible that an impact of low vision might be that readers are unable to fully develop phonological and orthographic strategies and this holds back their reading development.

Once an analysis of errors has been undertaken and has revealed any particular strategies being adopted, teaching methods can be adapted to respond to the findings. Again the field of deaf education may offer a useful example to consider in the area of low vision.

\section{References}

Conrad, R. (1979) The Deaf School Child. Harper and Row, London.

Corley, G. and Pring, L. (1993) The oral reading errors of partially sighted children. Br. J. Vis. Impair. 11, $24-27$.

Douglas, G., Grimley, M., Hill, E., Long, R. and Tobin, M. J. (2002) The use of the NARA for assessing the reading ability of children with low vision. Br. J. Vis. Impair. 20, 68-75.

Gompel, M., van Bon, W. H. J., Schreuder, R. and Andriaansen, J. J. M. (2002) Reading and spelling competence of Dutch children with low vision. J. Vis. Impair. Blind. 96, 435-447.

Goodman, K. S. (1967) Reading: a psycholinguistic guessing game. J. Read. Spec. 6, 126-135.

Goodman, K. S. (1969) Analysis of oral reading miscues: applied psycholinguistics. Read. Res. Q. 5, 9-30.

Laing, S. P. (2002) Miscue analysis in school-age children. Am. J. Speech Lang. Pathol. 11, 407-416.

Legge, G. E., Ahn, S. J., Klitz, T. S. and Luebker, A. (1996) Psychophysics of reading - XVI. The visual span in normal and low vision. Vision Res. 37, 1999-2009.

Leu, D. J. (1982) Oral reading errors analysis, a critical review of research and application. Read. Res. Q. 17, 420-437.

Neale, M. D. (1997) Neale Analysis of Reading Ability - Revised: Manual for Schools. NFER-Nelson, Windsor.

Nicholson, T., Lillas, M. and Rzoska, A. (1988) Have we been misled by miscues. Read. Teach. 42, 6-10.

Quigley, S. and Paul, P. V. (1990) Language and Deafness. Singular Publishing Co., San Diego, CA.

Southgate, V. (1962) Group Reading Test (Test 2, Form A - Sentence Completion). Hodder and Stoughton, Sevenoaks.

Tobin, M. J. (1993) The educational implications of visual impairment. In: The Management of Visual Impairment in Childhood (eds A. R. Fielder, A. B. Best and M. C. O. Bax), London: Mac Keith Press, 143149.

Webster, A. (1986) Deafness, Development and Literacy. Methuen, London.

Webster, A., Wood, D. and Griffiths, A. J. (1981) Reading retardation or linguistic deficit? 1: Interpreting reading test performances of hearing-impaired adolescents. J. Res. Read. 4, 136-147.

Wood, D. Griffiths, A. J. and Webster, A. (1981) Reading retardation or linguistic deficit? 11: test-answering strategies in hearing and hearing-impaired school children. J. Res. Read. 4, 148-157. 